\title{
A Note on Asymmetric Thick Branes
}

\author{
D. Bazeia, ${ }^{1,2}$ R. Menezes, ${ }^{2,3}$ and R. da Rocha ${ }^{4}$ \\ ${ }^{1}$ Departamento de Física, Universidade Federal da Paraíba, 58051-970 João Pessoa, PB, Brazil \\ ${ }^{2}$ Departamento de Física, Universidade Federal de Campina Grande, 58109-970 Campina Grande, PB, Brazil \\ ${ }^{3}$ Departamento de Ciências Exatas, Universidade Federal da Paraíba, 58297-000 Rio Tinto, PB, Brazil \\ ${ }^{4}$ CMCC, Universidade Federal do ABC, 09210-170 Santo André, SP, Brazil
}

Correspondence should be addressed to D. Bazeia; dbazeia@gmail.com

Received 4 April 2014; Revised 20 May 2014; Accepted 22 May 2014; Published 11 June 2014

Academic Editor: Rong-Gen Cai

Copyright (c) 2014 D. Bazeia et al. This is an open access article distributed under the Creative Commons Attribution License, which permits unrestricted use, distribution, and reproduction in any medium, provided the original work is properly cited. The publication of this article was funded by SCOAP S $^{3}$

\begin{abstract}
We study asymmetric thick braneworld scenarios, generated after adding a constant to the superpotential associated with the scalar field. We study in particular models with odd and even polynomial superpotentials, and we show that asymmetric brane can be generated irrespective of the potential being symmetric or asymmetric. We study in addition the nonpolynomial sine-Gordon like model, also constructed with the inclusion of a constant in the standard superpotential, and we investigate gravitational stability of the asymmetric brane. The results suggest robustness of the new braneworld scenarios and add further possibilities of the construction of asymmetric branes.
\end{abstract}

\section{Introduction}

The concept of braneworld with a single extra dimension of infinite extent [1] plays a fundamental role in high energy physics and cosmology. In such braneworld scenario, the particles of the standard model are confined to the brane, while the graviton can propagate in the whole space [1-6]; see also [7-14] for further details.

Most braneworld scenarios assume a $\mathbb{Z}_{2}$-symmetric brane, as motivated by the Horava-Witten model $[15,16]$. Notwithstanding, more general models that are not directly derived from $\mathrm{M}$-theory are obtained by relaxing the mirror symmetry across the brane [17-40]. Asymmetric branes are usually considered in the literature as a framework to models where the $\mathbb{Z}_{2}$ symmetry is not required. The parameters in the bulk, such as the gravitational and cosmological constants, can differ on either side of the brane. The term coined "asymmetric" branes means also that the gravitational parameters of the theory can differ on either side of the brane. In some cases, the Planck scale on the brane differs on either side of the domain wall [39-41]. One of the prominent features regarding asymmetric braneworld models is that they present some self-accelerating solutions without the necessity to consider an induced gravity term in the brane action [33].

Braneworld models without mirror symmetry have been investigated in further aspects. For instance, different black hole masses were obtained on the two sides on the brane [17], as well as different cosmological constants on the two sides [19-24]. In addition, one side of the brane can be unstable and the other one stable $[35,36]$. If the $\mathbb{Z}_{2}$ symmetry is taken apart, it is also possible to get infrared modifications of gravity, comprehensively considered in the asymmetric models in $[39,40]$ and in the hybrid asymmetric DGP model in [30] as well. In both models, the Planck mass and the cosmological constant are different at each side of the brane. In the asymmetric model, there is a hierarchy between the Planck masses and the AdS curvature scales on each side of the brane [34].

Asymmetric braneworlds can be further described by thick domain walls in a geometry that asymptotically is led to different cosmological constants, being de Sitter (dS) in one side and anti-de Sitter (AdS) in the other one, along the perpendicular direction to the wall. The asymmetry regarding the braneworld arises as the scalar field interpolates between two nondegenerate minima of a scalar potential without $\mathbb{Z}_{2}$ 
symmetry. Asymmetric static double-braneworlds with two different walls were also considered in this context, embedded in an $\mathrm{AdS}_{5}$ bulk. Furthermore, an independent derivation of the Friedmann equation was presented in the simplest form for an $\mathrm{AdS}_{5}$ bulk, with different cosmological constants on the two sides of the brane [38]. Finally, a braneworld that acts as a domain wall between two different five-dimensional bulks was considered as a solution to Gauss-Bonnet gravity [19, 33]. Models with moving branes have been further considered [42] in order to break the reflection symmetry of the brane model [17, 20, 22, 23, 43-45]. Other interesting models that deal with asymmetric branes have been done; some of them can be found in [46-51].

In the current work, we will further study asymmetric branes, focusing on general features, which we believe can be used to better qualify the braneworld scenario as symmetric or asymmetric. The key issue is to add a constant to the superpotential that describes the scalar field, which affects all the braneworld results. In particular, one notes that it explicitly alters the quantum potential that responds for stability, evincing, and unraveling prominent physical features, as the asymmetric localization of the graviton zero mode. We analyze all the abovementioned features of asymmetric branes in the context of superpotentials containing odd and even power in the scalar field, up to the third- and fourthorder power, respectively, and the sine-Gordon model.

The investigation starts with a brief revision of the equations that govern the braneworld scenario under investigation, getting to the first-order framework, where firstorder differential equations solve the Einstein and field equations if the potential has a very specific form. We deal with asymmetric branes, in the scenario where the brane is generated from a kink of two distinct and wellknown models, with the superpotentials being odd and even, respectively. Subsequently, the sine-Gordon model is also studied and the associated linear stability is investigated. The quantum potential and the graviton asymmetric zero mode are explicitly constructed.

\section{The Framework}

We start with a five-dimensional action in which gravity is coupled to a scalar field in the form

$$
\mathcal{S}=\int d^{5} x \sqrt{|g|}\left(-\frac{1}{4} R+\mathscr{L}\right),
$$

where

$$
\mathscr{L}=\frac{1}{2} \nabla_{a} \phi \nabla^{a} \phi-V(\phi)
$$

and $a=0,1, \ldots, 4$. We are using $4 \pi G=1$, with field and space and time variables dimensionless, for simplicity. By denoting $V_{\phi}=d V / d \phi$, the Einstein equations and the Euler-Lagrange equation are $G_{a b}=T_{a b}$ and $\nabla^{a} \nabla_{a} \phi+V_{\phi}=0$, respectively. We write the metric as

$$
d s^{2}=e^{2 A} \eta_{\mu \nu} d x^{\mu} d x^{\nu}-d y^{2}
$$

where $A=A(y)$ describes the warp function and only depends on the extra dimension $y$. Taking the scalar field with same dependence, we obtain

$$
\begin{gathered}
A^{\prime \prime}+\frac{2}{3} \phi^{\prime 2}=0, \\
A^{\prime 2}-\frac{1}{6} \phi^{\prime 2}+\frac{1}{6} V(\phi)=0, \\
\phi^{\prime \prime}+4 \phi^{\prime} A^{\prime}-V_{\phi}=0,
\end{gathered}
$$

where prime stands for derivative with respect to the extra dimension. As one knows, solutions to the first-order equations,

$$
\begin{gathered}
\phi^{\prime}=\frac{1}{2} W_{\phi}, \\
A^{\prime}=-\frac{1}{3} W(\phi),
\end{gathered}
$$

also solve (4a), (4b), and (4c), if the potential has the following form:

$$
V=\frac{1}{8} W_{\phi}^{2}-\frac{1}{3} W^{2}
$$

where $W=W(\phi)$ is a function of the scalar field $\phi$. This result shows that if one adds a constant to $W$, it will modify the potential and, consequently, all the results that follow from the model.

To better explore this idea, we study three distinct models, which generalize previous investigations.

2.1. The Case of an Odd Superpotential. Let us introduce the function

$$
W_{c}(\phi)=-\frac{2}{3} \phi^{3}+2 \phi+c
$$

where $c$ is a real constant. This $W$ is an odd function for $c=0$. The potential given by (6) has now the form

$$
V(\phi)=\frac{1}{2}\left(1-\phi^{2}\right)^{2}-\frac{1}{3}\left(c+2 \phi-\frac{2}{3} \phi^{3}\right)^{2} .
$$

It has the $\mathbb{Z}_{2}$ symmetry only when $c=0$; the symmetry is now $\phi \mapsto-\phi$ and $c \mapsto-c$. There are minima at $\phi_{ \pm}= \pm 1$, with

$$
W_{c}\left(\phi_{ \pm}\right)= \pm \frac{4}{3}+c
$$

The maxima depend on $c$ and are solutions of the algebraic equation $4 \phi_{\max }^{3}-39 \phi_{\max }-2 c=0$; see Figure 1 .

We focus attention on the scalar field. There is a topological sector connecting the minima. In this sector, there are two solutions (kink and antikink), which can be mapped to one another when $\phi \mapsto-\phi$ or $y \mapsto-y$. Thus, we study solely the kink like solution. The first-order equation for the field 


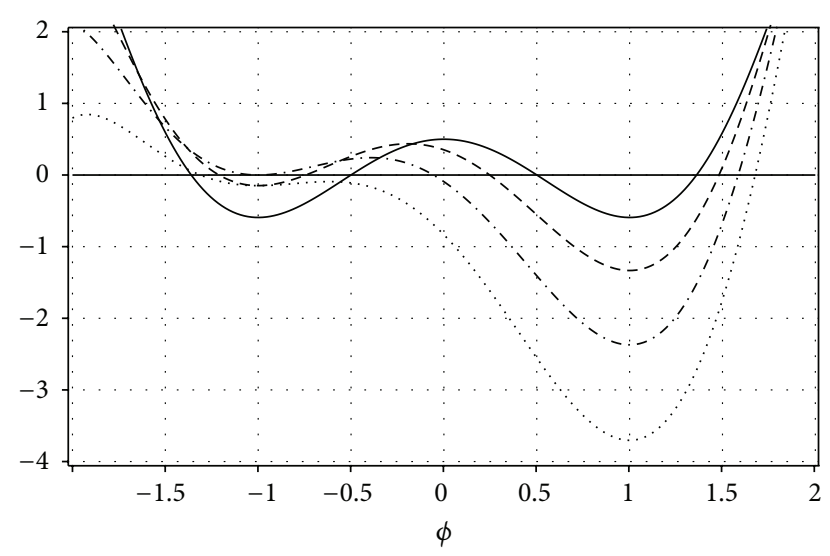

FIGURE 1: The potential given by (8) for $c=0$ (black line), $c=2 / 3$ (dashed curve), $c=4 / 3$ (dot-dashed line), and $c=2$ (dotted line).

does not depend on the parameter $c$ and is provided by $\phi^{\prime}=$ $1-\phi^{2}$. The solution for this equation is

$$
\phi(y)=\tanh (y),
$$

with $\phi( \pm \infty)=\phi_{ \pm}= \pm 1$. It obeys $\phi(y)=-\phi(-y)$, and it connects symmetric minima.

The warp function can be obtained by using (5b):

$$
A(y)=-\frac{1}{9} \tanh ^{2}(y)+\frac{4}{9} \ln (\operatorname{sech}(y))-\frac{c}{3} y,
$$

with $A(0)=0$. We note that the behavior of this function outside the brane can be written as

$$
A_{ \pm \infty}(y)=-\frac{1}{3} W\left(\phi_{ \pm}\right)|y|=-\frac{1}{3}\left(\frac{4}{3} \pm c\right)|y|
$$

and asymptotically the five-dimensional cosmological constant is

$$
\Lambda_{5_{ \pm}} \equiv V(\phi( \pm \infty))=-\frac{1}{3}\left(\frac{4}{3} \pm c\right)^{2} .
$$

If $c=0$, the brane is symmetric, connecting two regions in the $\mathrm{AdS}_{5}$ bulk, with the same cosmological constant $\Lambda_{5_{+}}=-16 / 27$. If $|c|>4 / 3$, the warp factor $e^{2 A}$ diverges. In all other cases, the warp factor describes asymmetric braneworlds. If $|c|=4 / 3$, the brane connects an $\mathrm{AdS}_{5}$ bulk and a five-dimensional Minkowski $\left(\mathbb{M}_{5}\right)$ bulk, with $\Lambda_{5+}=$ $-64 / 27\left[\Lambda_{5+}=0\right]$ and $\Lambda_{5_{-}}=0\left[\Lambda_{5-}=-64 / 27\right]$, when $c=4 / 3[c=-4 / 3]$. If $0<|c|<4 / 3$, the brane connects two distinct $\mathrm{AdS}_{5}$ bulk spaces.

In Figure 2, we plot the profile of the warp factor, for some values of $c$. The solid curve represents the symmetric profile (Brane I). The dashed curve represents the asymmetric brane that connects two $\mathrm{AdS}_{5}$ bulk spaces, with $c=2 / 3$, $\Lambda_{5+}=-4 / 3$, and $\Lambda_{5-}=-4 / 27$ (Brane II). The dot-dashed curve represents the case where the brane connects an $\mathrm{AdS}_{5}$ to a $\mathbb{M}_{5}$ bulk (Brane III). Finally, the dotted curve represents the divergent warp factor with $c=2$.

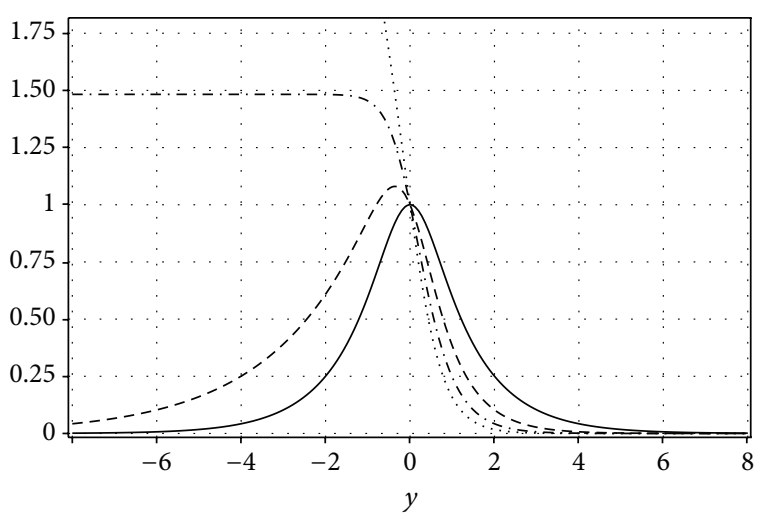

Figure 2: The warp factor $e^{2 A}$ with $A$ given by (11) for $c=0$ (solid line), $c=2 / 3$ (dashed line), $c=4 / 3$ (dot-dashed line), and $c=2$ (dotted line). We fix $A(0)=0$.

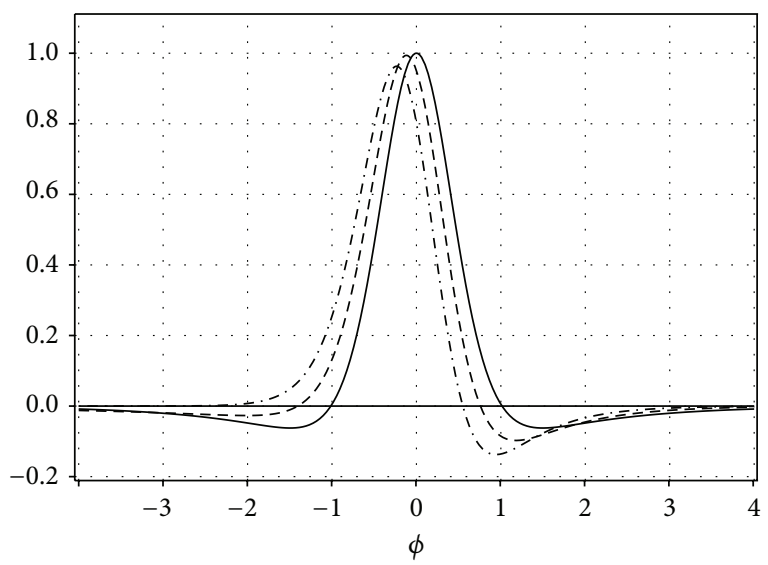

FIGURE 3: The energy density given by (14) for $c=0$ (solid line), $c=2 / 3$ (dashed line), and $c=4 / 3$ (dot-dashed line).

The energy density is given by

$$
\rho(y)=-e^{2 A} \mathscr{L},
$$

and it also depends on $c$ :

$$
\begin{gathered}
\rho(y)=e^{2 A}\left(c^{2}-\frac{16}{81}+\frac{4}{27} \operatorname{sech}^{4}(y)+\frac{31}{81} \operatorname{sech}^{6}(y)\right. \\
\left.-\frac{4 c}{27} \tanh (y)\left(2+\operatorname{sech}^{2}(y)\right)\right) .
\end{gathered}
$$

It is symmetric only for $c=0$. When $c \neq 0$, there exist contributions to the asymmetry from both the warp factor $e^{2 A}$ and the Lagrange density $\mathscr{L}$. In Figure 3 , we depict the profile for Branes I (solid curve), II (dashed curve), and III (dot-dashed curve). Therefore, for $c \neq 0$, the warp factor and the energy density are asymmetric.

This model shows that, although the kink like solution connects symmetric minima, the potential is asymmetric and gives rise to braneworld scenario which is also asymmetric, unless $c=0$. 


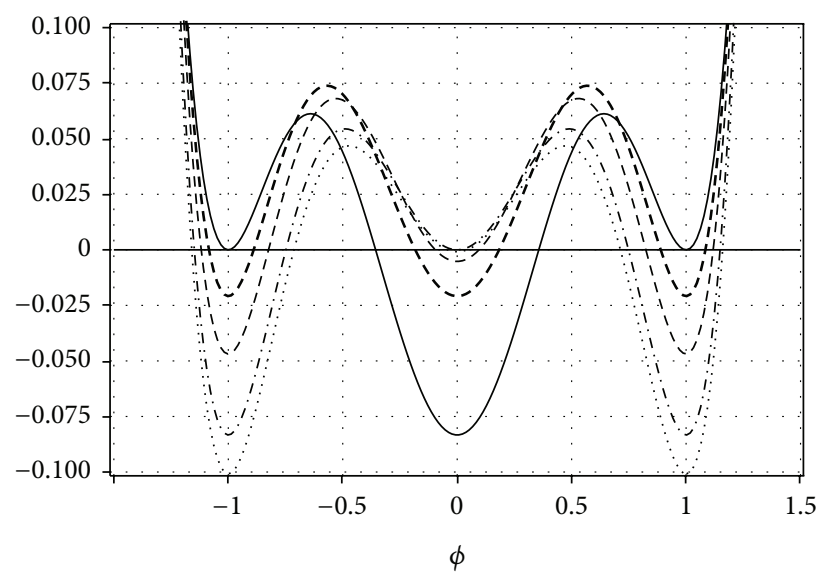

Figure 4: The potential given by (16) for $c=-1 / 2$ (solid line), $c=$ $-1 / 4$ (long dashed line), $c=-1 / 8$ (dashed line), $c=0$ (dot-dashed line), and $c=1 / 20$ (dotted line).

2.2. The Case of an Even Superpotential. Let us now investigate a different model, which presents kink like solution that connects asymmetric minima. It is defined by

$$
W_{c}(\phi)=-\frac{1}{2} \phi^{4}+\phi^{2}+c .
$$

This $W$ is an even function of $\phi$. The potential given by (6) is now

$$
V(\phi)=\frac{1}{2} \phi^{2}\left(1-\phi^{2}\right)^{2}-\frac{1}{3}\left(c+\phi^{2}-\frac{1}{2} \phi^{4}\right)^{2} .
$$

It is depicted in Figure 4, and it has the $\mathbb{Z}_{2}$ symmetry for any value of the parameter $c$. The minima are $\phi_{-}=-1, \phi_{0}=0$, and $\phi_{+}=1$, with

$$
W_{c}\left(\phi_{ \pm}\right)=\frac{1}{2}+c, \quad W_{c}\left(\phi_{0}\right)=c .
$$

There are two topological sectors. The first connects the minima $\phi_{-}$and $\phi_{0}$, while the second connects the minima $\phi_{0}$ and $\phi_{+}$. Note that these sectors can be mapped by taking the transformation $\phi \mapsto-\phi$. In each sector, there are two solutions (kink and antikink); they can be mapped into each other with the coordinate transformation $y \mapsto-y$. Thus, we only study one of these solutions. Using the first-order equation, $\phi^{\prime}=\phi\left(1-\phi^{2}\right)$, we find the solution

$$
\phi(y)=\frac{\sqrt{2}}{2} \sqrt{1+\tanh (y)}
$$

which connects asymmetric minima.

Here, the warp function is given by

$$
A(y)=-\frac{1}{24} \tanh (y)+\frac{1}{12} \ln (\operatorname{sech}(y))-\left(\frac{1}{12}+\frac{c}{3}\right) y
$$

and presents the asymptotic values

$$
A_{ \pm \infty}(y)=-\frac{1}{3}\left[\frac{1}{4} \pm\left(\frac{1}{4}+c\right)\right]|y|
$$

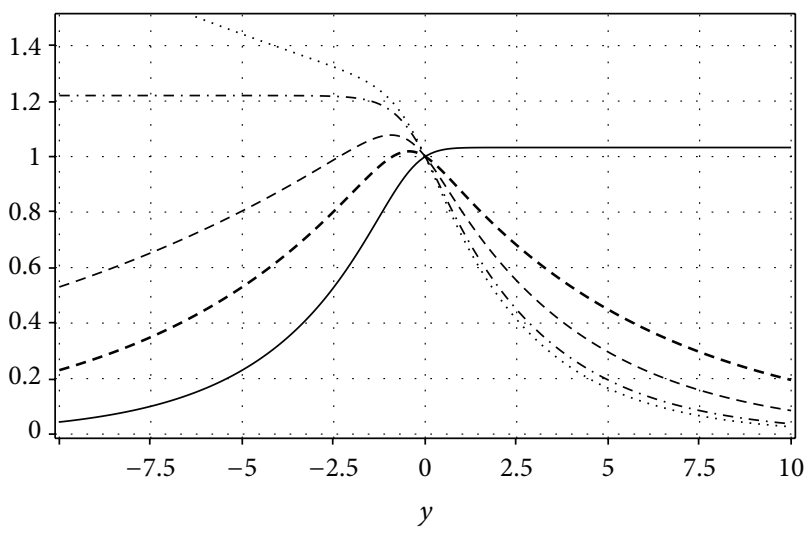

FIgURE 5: The warp factor $e^{2 A}$ with $A$ given by (19) for $c=-1 / 2$ (solid line), $c=-1 / 4$ (long dashed line), $c=-1 / 8$ (dashed line), $c=0$ (dot-dashed line), and $c=1 / 20$ (dotted line).

The bulk cosmological constant is provided by

$$
\Lambda_{5_{ \pm}}=-\frac{1}{3}\left[\frac{1}{4} \pm\left(\frac{1}{4}+c\right)\right]^{2}
$$

For $c<-1 / 2$ and $c>0$, the warp factor diverges. We obtain the asymmetric branes, separating two bulk spaces $\mathbb{M}_{5}$ and $\mathrm{AdS}_{5}$, for $c=-1 / 2$ and $c=0$. In the first case, $\Lambda_{5+}=0$ and $\Lambda_{5-}=-1 / 12$, while in the second case $\Lambda_{5+}=-1 / 12$ and $\Lambda_{5-}=0$. The energy density is

$$
\begin{aligned}
\rho(y)=e^{2 A}\left[\frac{39}{64}-\frac{c}{12}-\frac{c^{2}}{9}-\left(\frac{31}{48}-\frac{c}{18}\right) \tanh (y)\right. \\
+\left(\frac{c}{36}-\frac{35}{288}\right) \tanh ^{2}(y)+\frac{17}{144} \tanh ^{3}(y) \\
\left.-\frac{1}{576} \tanh ^{4}(y)\right] .
\end{aligned}
$$

In Figures 5 and 6, we depict the warp factor and the energy density, for some values of $c$.

This model is different from the previous one. The potential is always symmetric, and the kink like solution connects asymmetric minima. The model gives rise to warp factor that is always asymmetric; although at $c=-1 / 4$ it connects similar $\mathrm{AdS}_{5}$ bulk spaces.

\section{Stability}

An issue of interest concerns stability of the gravity sector of the braneworld model. For the models studied in the previous section, such investigations can be implemented numerically [52], an issue to be described in the longer work under preparation [53]. Here, however, to gain confidence on the stability of the suggested braneworld scenarios, we develop the analytical procedure: we get inspiration from the first work in [7-14], where it is shown that the sine-Gordon model 


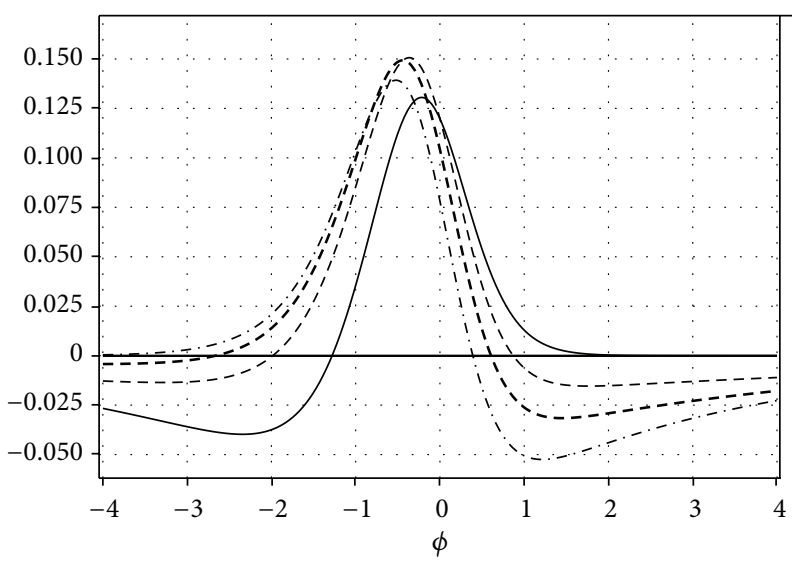

FIgURE 6: The energy density given by (21) for $c=-1 / 2$ (solid line), $c=-1 / 4$ (long dashed line), $c=-1 / 8$ (dashed line), and $c=0$ (dot-dashed line).

is good model for analytical investigation. Thus, we consider the sine-Gordon-type model with

$$
W_{c}(\phi)=2 \sqrt{\frac{3}{2}} \sin \left(\sqrt{\frac{3}{2}} \phi\right)+c
$$

with $|c| \leq \sqrt{6}$. We note that, for $\phi$ being small, the model is similar to the case of an odd superpotential studied previously; thus, the current investigation engenders results that are also valid for the model investigated in Section 2.1. The point here is that we want to study stability analytically, so the sine-Gordon model is the appropriate model.

We use (5a) and (5b) to obtain analytic solutions:

$$
\begin{gathered}
\phi(y)=\sqrt{\frac{3}{2}} \arcsin (\tanh (y)), \\
A_{c}(y)=A_{0}(y)-\frac{1}{3} c y, \\
A_{0}(y)=-\ln (\operatorname{sech}(y)),
\end{gathered}
$$

where $\phi(y)$ and $A_{0}(y)$ are the standard solutions of the sineGordon model, for $c=0$.

To study stability, we follow [52]. We work in the transverse traceless gauge to decouple gravity from the scalar field. Also, we have to change from $y$ to the conformal coordinate $z=z(y)$ to get to a Schroedinger like equation with a quantum mechanical potential. This investigation cannot be done analytically anymore, unless we take $c=0$. Thus, we resort to the approximation, taking $c$ very small and expanding the results up to first-order in $c$. In this case, we can write the conformal coordinate as

$$
\begin{gathered}
z(y)=f(y)+\frac{c}{3}\left(y f(y)-\int d y f(y)\right) \\
f(y)=\int d y e^{-A(y)}
\end{gathered}
$$

We note that $f(y)$ is an even function, while the $c$-term contribution is odd. Therefore $z(y)$ is an asymmetric function. The inverse is

$$
y=f^{-1}(z)-\frac{c}{3}\left[\frac{1}{f^{\prime}(x)}\left(x z-\int d x f(x)\right)\right]_{x=f^{-1}(z)} .
$$

After some algebraic calculations, we could find the associated potential

$$
U(z)=\frac{3}{2} A^{\prime \prime}+\frac{9}{4} A^{\prime 2}+c U_{c}(z),
$$

where $U_{c}(z)$ is the contribution up to first-order in $c$. The sineGordon-type model is nice, and we could find the explicit results: the conformal coordinate $z=\int e^{-A(y)} d y$ in (24a) is computed as

$$
z(y)=\sinh (y)+\frac{c}{3}(y \sinh (y)-\cosh (y)) .
$$

Since $c$ is small, the above expression can be inverted to give

$$
\begin{aligned}
y(z)=\operatorname{arcsinh}(z)-\frac{c}{3}[ & \frac{1}{\sqrt{1+(z)^{2}}} \\
& \left.\times\left((z) \operatorname{arcsinh}(z)-\sqrt{1+(z)^{2}}\right)\right] .
\end{aligned}
$$

Therefore,

$$
\begin{aligned}
A(z)= & -\frac{1}{2} \ln \left(1+(z)^{2}\right) \\
& -c\left(\frac{\operatorname{arcsinh}(z)}{1+(z)^{2}}+\frac{z}{\sqrt{1+(z)^{2}}}\right) .
\end{aligned}
$$

It follows from (26) that the quantum potential is written as

$$
\begin{gathered}
U(z)=\frac{3}{4} \frac{\left(5 z^{2}-2\right)}{\left(1+z^{2}\right)^{2}}+c U_{c}(z), \\
U_{c}(z)=\frac{1}{\left(1+z^{2}\right)^{3}}\left(\left(1-6 z^{2}\right) \operatorname{arcsinh}(z)\right. \\
\left.+7 z \sqrt{1+z^{2}}\right) .
\end{gathered}
$$

It is depicted in Figure 7. The maxima of the potential $U(z)$ are provided by

$$
\begin{aligned}
z_{ \pm} & = \pm \frac{3}{\sqrt{5}}-\frac{\sqrt{70} c}{9450}\left(27 \sqrt{5} \operatorname{arcsinh}\left(\frac{3}{\sqrt{5}}\right) \sqrt{70}-1330\right) \\
& \approx \pm 1.34164-0.68398 c .
\end{aligned}
$$




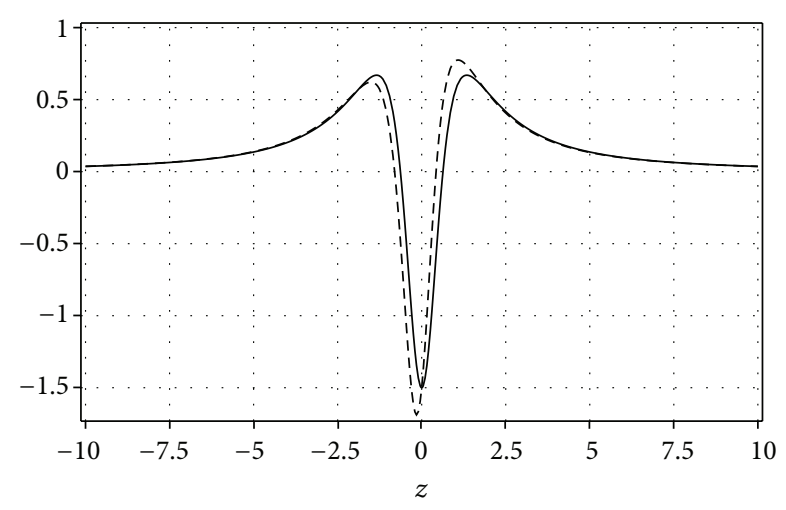

Figure 7: Plot of the quantum mechanical potential in (30a) and (30b), for $c=0$ (solid line) and $c=1 / 5$ (long dashed line).

In the limit $z \rightarrow \infty$, the asymptotic value of the potential reads

$$
U(z) \approx \frac{15}{4 z^{2}}-\frac{1}{z^{4}}(9+c(6 \ln (2 z)-7))+\mathcal{O}\left(\frac{1}{z^{6}}\right) .
$$

It explicitly shows how the asymmetric contribution enters the game asymptotically.

The quantum mechanical analogous problem is described by the equation

$$
\left(-\frac{d^{2}}{d z^{2}}+\widetilde{U}(z)\right) \psi_{n}=k^{2} \psi_{n}
$$

This Hamiltonian can be factorized as, up to first-order in $c$,

$$
-\frac{d^{2}}{d x^{2}}+U(x)=\left(-\frac{d}{d x}+\frac{3}{2} A^{\prime}\right)\left(\frac{d}{d x}+\frac{3}{2} A^{\prime}\right),
$$

where $A=A(z)$ is given by (29). It is then nonnegative, so there are no negative bound states. In fact, the graviton zero mode is $\psi_{0}(z)$, which solves the above equation for $k=0$; it is given explicitly by

$$
\begin{aligned}
\psi_{0}(z)= & \exp \left(\frac{3}{2} A(z)\right) \\
= & \left(1+(z)^{2}\right)^{-3 / 4} \\
& \times\left(1-\frac{c}{3}\left(\frac{\operatorname{arcsinh}(z)}{1+z^{2}}+\frac{z}{\sqrt{1+z^{2}}}\right)\right)
\end{aligned}
$$

and is depicted in Figure 8, showing its asymmetric behavior. It has no node, indicating that it is the lowest bound state, as expected.

\section{Concluding Remarks}

In this work, we investigated the presence of asymmetric branes, constructed from the addition of a constant $c$ to $W(\phi)$, the function that defines the potential in the form

$$
V(\phi)=\frac{1}{8} W_{\phi}^{2}-\frac{1}{3} W^{2} .
$$

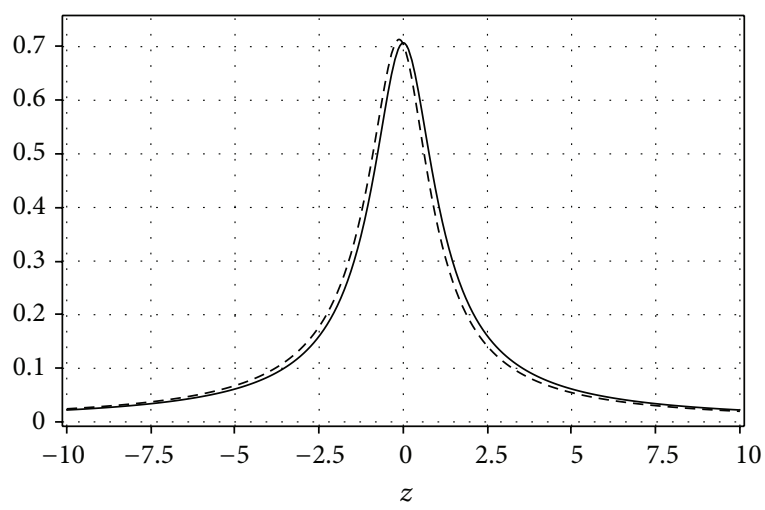

FIGURE 8: Plot of the normalized graviton zero mode $\psi_{0}(z)$, for $c=0$ (solid line) and for $c=1 / 5$ (long dashed line).

This expression controls the scalar field model that generates the thick brane scenario, and it is important to reduce the equations to its first-order form:

$$
\phi^{\prime}=\frac{1}{2} W_{\phi} ; \quad A^{\prime}=-\frac{1}{3} W
$$

The main results of the current work show that we can construct asymmetric brane, irrespective of the scalar potential being symmetric or asymmetric.

We have investigated two distinct models, of the $\phi^{4}$ and $\phi^{6}$ type, and we have explicitly shown how the constant added to $W(\phi)$ works to induce asymmetry to the thick braneworld scenario. Moreover, we also studied the sine-Gordon-type model, focusing on the stability of the gravitational scenario. The nice properties of the sine-Gordon model very much helped us to implement analytical calculations, showing stability of the graviton zero mode, despite the presence of an asymmetric volcano potential.

Evidently, the asymmetry induced in the thick braneworld scenario may be phenomenologically relevant, since it has to be consistent with the $\mathrm{AdS}_{5}$ bulk curvature and to the experimental and theoretical limits of the brane thickness $[54,55]$.

When finishing this study, we become aware of the work [56], which investigates similar possibilities.

\section{Conflict of Interests}

The authors declare that there is no conflict of interests regarding the publication of this paper.

\section{Acknowledgments}

The authors would like to thank CAPES and CNPq for partial financial support.

\section{References}

[1] L. Randall and R. Sundrum, "An alternative to compactification," Physical Review Letters, vol. 83, no. 23, pp. 4690-4693, 1999. 
[2] W. D. Goldberger and M. B. Wise, "Modulus stabilization with bulk fields," Physical Review Letters, vol. 83, pp. 4922-4925, 1999.

[3] O. DeWolfe, D. Z. Freedman, S. S. Gubser, and A. Karch, "Modeling the fifth dimension with scalars and gravity," Physical Review D: Particles, Fields, Gravitation and Cosmology, vol. 62, no. 4, Article ID 046008, pp. 1-16, 2000.

[4] C. Csáki, J. Erlich, T. J. Hollowood, and Y. Shirman, "Universal aspects of gravity localized on thick branes," Nuclear Physics B, vol. 581, no. 1-2, pp. 309-338, 2000.

[5] C. Csáki, J. Erlich, C. Grojean, and T. J. Hollowood, “General properties of the self-tuning domain wall approach to the cosmological constant problem," Nuclear Physics B, vol. 584, no. 1-2, pp. 359-386, 2000.

[6] C. Csaki, "TASI lectures on extra dimensions and branes," http://arxiv.org/abs/hep-ph/0404096.

[7] M. Gremm, "Four-dimensional gravity on a thick domain wall," Physics Letters B, vol. 478, no. 4, pp. 434-438, 2000.

[8] F. A. Brito, M. Cvetic, and S. C. Yoon, "From a thick to a thin supergravity domain wall," Physical Review D, vol. 64, Article ID 064021, 2001.

[9] M. Cvetič and N. D. Lambert, "Effective supergravity for supergravity domain walls," Physics Letters B, vol. 540, no. 3-4, pp. 301-308, 2002.

[10] A. Campos, "Critical phenomena of thick branes in warped spacetimes," Physical Review Letters, vol. 88, no. 14, Article ID 141602, 4 pages, 2002.

[11] A. Melfo, N. Pantoja, and A. Skirzewski, “Thick domain wall spacetimes with and without reflection symmetry," Physical Review D, vol. 67, no. 10, Article ID 105003, 6 pages, 2003.

[12] D. Bazeia, F. A. Brito, and J. R. Nascimento, "Supergravity brane worlds and tachyon potentials," Physical Review D, vol. 68, no. 8, 6 pages, 2003.

[13] D. Bazeia, C. Furtado, and A. R. Gomes, "Brane structure from a scalar field in warped spacetime," Journal of Cosmology and Astroparticle Physics, no. 2, article 002, 9 pages, 2004.

[14] D. Bazeia and A. R. Gomes, "Bloch brane," Journal of High Energy Physics, vol. 2004, no. 5, article 012, 2004.

[15] P. Hořava and E. Witten, "Heterotic and type I string dynamics from eleven dimensions," Nuclear Physics B, vol. 460, no. 3, pp. 506-524, 1996.

[16] P. Hořava and E. Witten, "Eleven-dimensional supergravity on a manifold with boundary," Nuclear Physics B, vol. 475, no. 1-2, pp. 94-114, 1996.

[17] P. Kraus, "Dynamics of anti-de Sitter domain walls," Journal of High Energy Physics, vol. 1999, no. 9912, article 011, 1999.

[18] D. Ida, "Brane-world cosmology," Journal of High Energy Physics, vol. 2000, no. 0009, article 014, 2000.

[19] N. Deruelle and T. Doležel, "Brane versus shell cosmologies in Einstein and Einstein-Gauss-Bonnet theories," Physical Review D, vol. 62, no. 10, Article ID 103502, 8 pages, 2000.

[20] W. B. Perkins, "Colliding bubble worlds," Physics Letters B, vol. 504, no. 1-2, pp. 28-32, 2001.

[21] P. Bowcock, C. Charmousis, and R. Gregory, "General brane cosmologies and their global spacetime structure," Classical and Quantum Gravity, vol. 17, no. 22, pp. 4745-4763, 2000.

[22] B. Carter, J.-P. Uzan, R. A. Battye, and A. Mennim, "Simulated gravity without true gravity in asymmetric brane-world scenarios," Classical and Quantum Gravity, vol. 18, no. 22, pp. 48714895, 2001.
[23] H. Stoica, S.-H. H. Tye, and I. Wasserman, "Cosmology in the Randall-Sundrum brane world scenario," Physics Letters B, vol. 482, no. 1-3, pp. 205-212, 2000.

[24] L. Á. Gergely, "Generalized Friedmann branes," Physical Review D. Third Series, vol. 68, no. 12, Article ID 124011, 13 pages, 2003.

[25] S. A. Appleby and R. A. Battye, "Regularized braneworlds of arbitrary codimension," Physical Review D: Particles, Fields, Gravitation, and Cosmology, vol. 76, no. 12, Article ID 124009, 16 pages, 2007.

[26] G. Konas, "How to go with an RG flow," Journal of High Energy Physics, vol. 2001, no. 08, article 041, 2001.

[27] H. Collins and R. Holdom, "Brane cosmologies without orbifolds," Physical Review D, vol. 62, Article ID 105009, 2000.

[28] Yu. V. Shtanov, “Closed system of equations on a brane," Physics Letters B, vol. 541, no. 1-2, pp. 177-182, 2002.

[29] Y. Shtanov, A. Viznyuk, and L. N. Granda, "Asymmetric embedding in brane cosmology," Modern Physics Letters A: Particles and Fields, Gravitation, Cosmology, Nuclear Physics, vol. 23, no. 12, pp. 869-878, 2008.

[30] C. Charmousis, R. Gregory, and A. Padilla, "Stealth acceleration and modified gravity," Journal of Cosmology and Astroparticle Physics, vol. 2007, no. 10, article 006, 2007.

[31] K. Koyama and K. Koyama, "Brane-induced gravity from asymmetric warped compactification," Physical Review D, vol. 72, Article ID 043511, 2005.

[32] H. Zhang, Z.-K. Guo, C. Chen, and X.-Z. Li, "On asymmetric brane creation," Journal of High Energy Physics, vol. 2012, article 19, 2012.

[33] K. Nozari and T. Azizi, "Phantom-like effects in an asymmetric brane embedding with induced gravity and the Gauss-Bonnet term in the bulk," Physica Scripta, vol. 83, Article ID 015001, 2011.

[34] E. O'Callaghan, R. Gregory, and A. Pourtsidou, “The cosmology of asymmetric Brane modified gravity," Journal of Cosmology and Astroparticle Physics, vol. 2009, no. 0909, article 020, 2009.

[35] Y. Shtanov, V. Sahni, A. Shaeloo, and A. Toporensky, "Induced cosmological constant and other features of asymmetric brane embedding," Journal of Cosmology and Astroparticle Physics, vol. 2009, no. 0904, article 023, 2009.

[36] K. Koyama, A. Padilla, and F. P. Silva, "Ghosts in asymmetric brane gravity and the decoupled stealth limit," Journal of High Energy Physics, vol. 2009, no. 3, article 134, 19 pages, 2009.

[37] R. Guerrero, R. O. Rodriguez, and R. S. Torrealba, "de Sitter and double asymmetric brane worlds," Physical Review D, vol. 72, Article ID 124012, 2005.

[38] L. Á. Gergely and R. Maartens, "Asymmetric brane-worlds with induced gravity," Physical Review D, vol. 71, no. 2, Article ID 024032, 7 pages, 2005.

[39] A. Padilla, "Cosmic acceleration from asymmetric branes," Classical and Quantum Gravity, vol. 22, no. 4, pp. 681-694, 2005.

[40] A. Padilla, "Infra-red modification of gravity from asymmetric branes," Classical and Quantum Gravity, vol. 22, pp. 1087-1104, 2005.

[41] R. Gregory and A. Padilla, "Nested braneworlds and strong brane gravity," Physical Review D, vol. 65, no. 8, Article ID 084013, 4 pages, 2002.

[42] A. Kehagias and E. Kiritsis, "Mirage cosmology," Journal of High Energy Physics, vol. 1999, no. 11, article 022, 1999.

[43] J. P. Uzan, "Asymmetric brane-world scenarios and simulated gravity," International Journal of Theoretical Physics, vol. 41, no. 11, pp. 2299-2309, 2002. 
[44] R. A. Battye, B. Carter, A. Mennim, and J.-P. Uzan, "Einstein equations for an asymmetric brane-world," Physical Review D. Third Series, vol. 64, no. 12, Article ID 124007, 19 pages, 2001.

[45] A.-C. Davis, S. Davis, W. B. Perkins, and I. R. Vernon, "Cosmological phase transitions in a brane world," Physical Review D, vol. 63, Article ID 083518, 2001.

[46] R. Guerrero, R. O. Rodriguez, R. Torrealba, and R. Ortiz, "De Sitter and double irregular domain walls," General Relativity and Gravitation, vol. 38, no. 5, pp. 845-855, 2006.

[47] A. de Souza Dutra, A. C. Amaro de Faria Jr., and M. Hott, "Degenerate and critical Bloch branes," Physical Review D: Particles, Fields, Gravitation, and Cosmology, vol. 78, no. 4, Article ID 043526, 9 pages, 2008.

[48] Z. H. Zhao, Y. X. Liu, and H. T. Li, "Fermion localization on asymmetric two-field thick branes," Classical and Quantum Gravity, vol. 27, no. 18, Article ID 185001, 2010.

[49] Y. X. Liu, C. E. Fu, L. Zhao, and Y. S. Duan, "Localization and mass spectra of fermions on symmetric and asymmetric thick branes," Physical Review D, vol. 80, Article ID 065020, 2009.

[50] Y.-X. Liu, Z.-H. Zhao, S.-W. Wei, and Y.-S. Duan, "Bulk matters on symmetric and asymmetric de Sitter thick branes," Journal of Cosmology and Astroparticle Physics, vol. 2009, no. 02, article 003, 2009.

[51] Q.-Y. Xie, J. Yang, and L. Zhao, "Resonance mass spectra of gravity and fermion on Bloch," Physical Review D, vol. 88, Article ID 105014, 2013.

[52] D. Bazeia, A. R. Gomes, and L. Losano, "Gravity localization on thick branes: a numerical approach," International Journal of Modern Physics A, vol. 24, no. 6, pp. 1135-1160, 2009.

[53] D. Bazeia, L. Losano, R. Menezes, and R. da Rocha, "Study of models of the sine-Gordon type in flat and curved spacetime," European Physical Journal C, vol. 73, no. 7, pp. 1-8, 2013.

[54] D. J. Kapner, T. S. Cook, E. G. Adelberger et al., "Tests of the gravitational inverse-square law below the dark-energy length scale," Physical Review Letters, vol. 98, Article ID 021101, 2007.

[55] J. M. Hoff da Silva and R. D. Rocha, "Effective monopoles within thick branes," EPL, vol. 100, no. 1, Article ID 11001, 2012.

[56] A. Ahmed, L. Dulny, and B. Grzadkowski, "Generalized Randall-Sundrum model with a single thick brane," European Physical Journal C, vol. 74, Article ID 2862, 18 pages, 2014. 

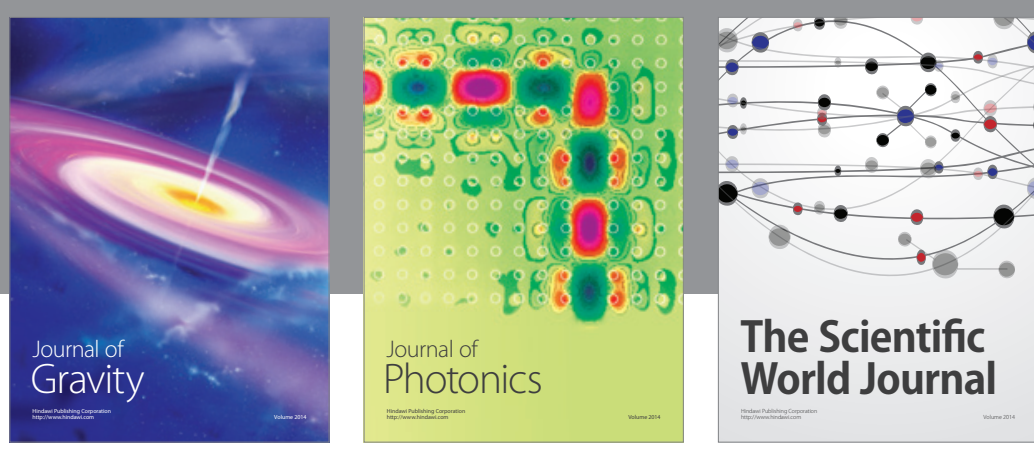

The Scientific World Journal
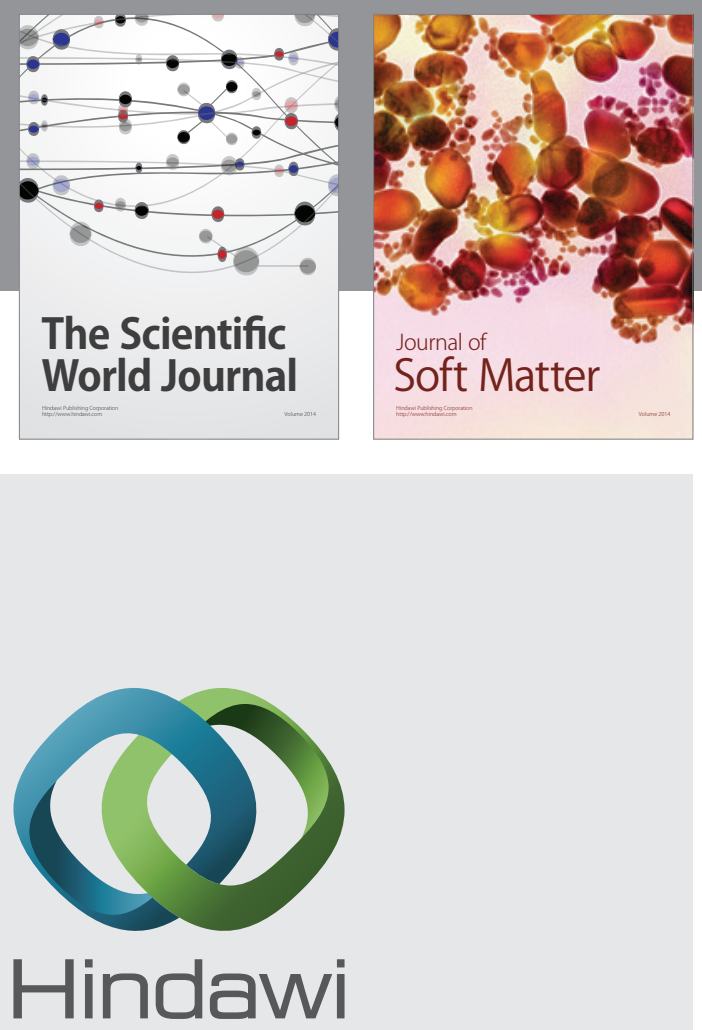

Submit your manuscripts at

http://www.hindawi.com

nternational Journal of

Statistical Mechanics
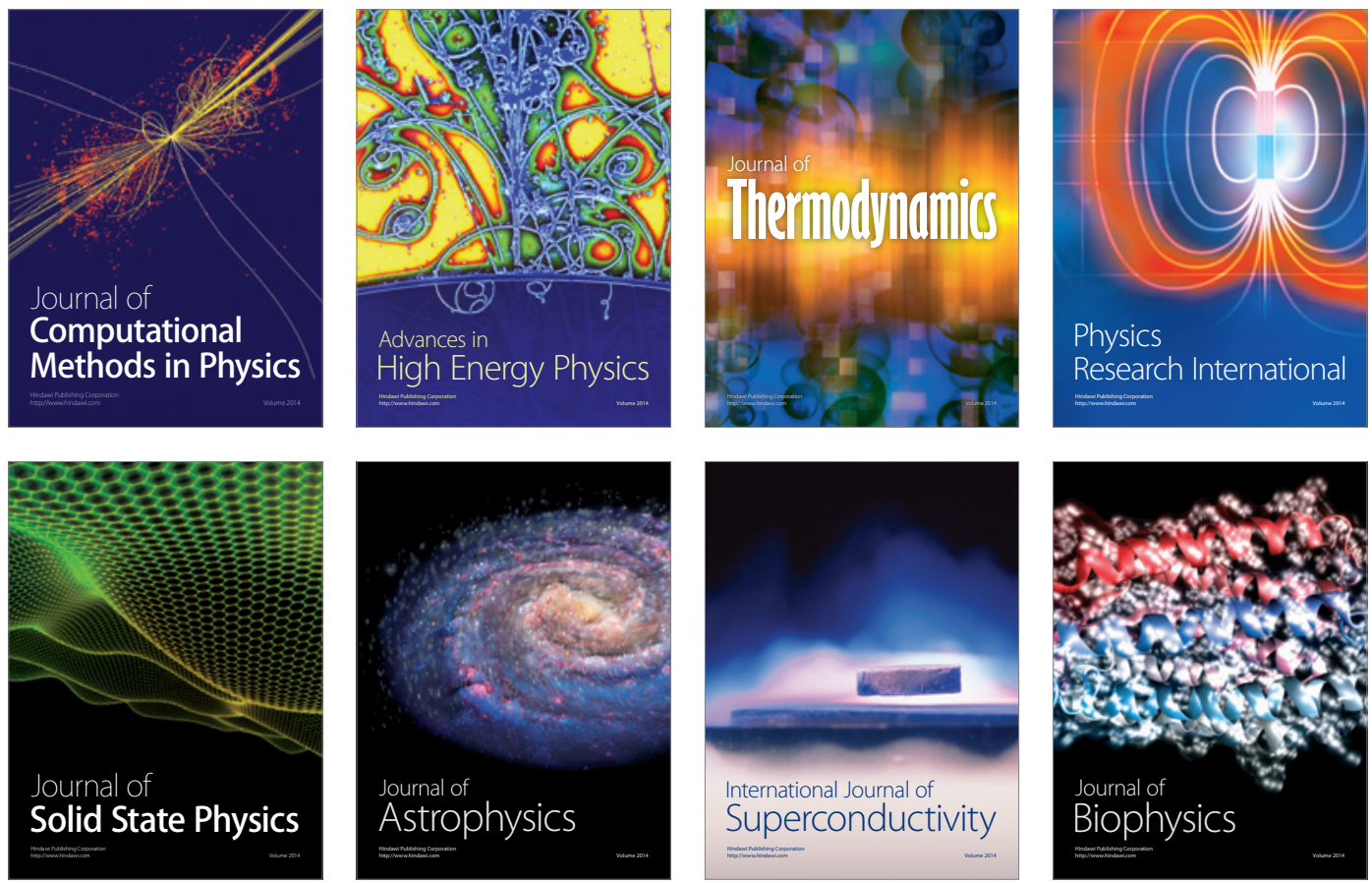
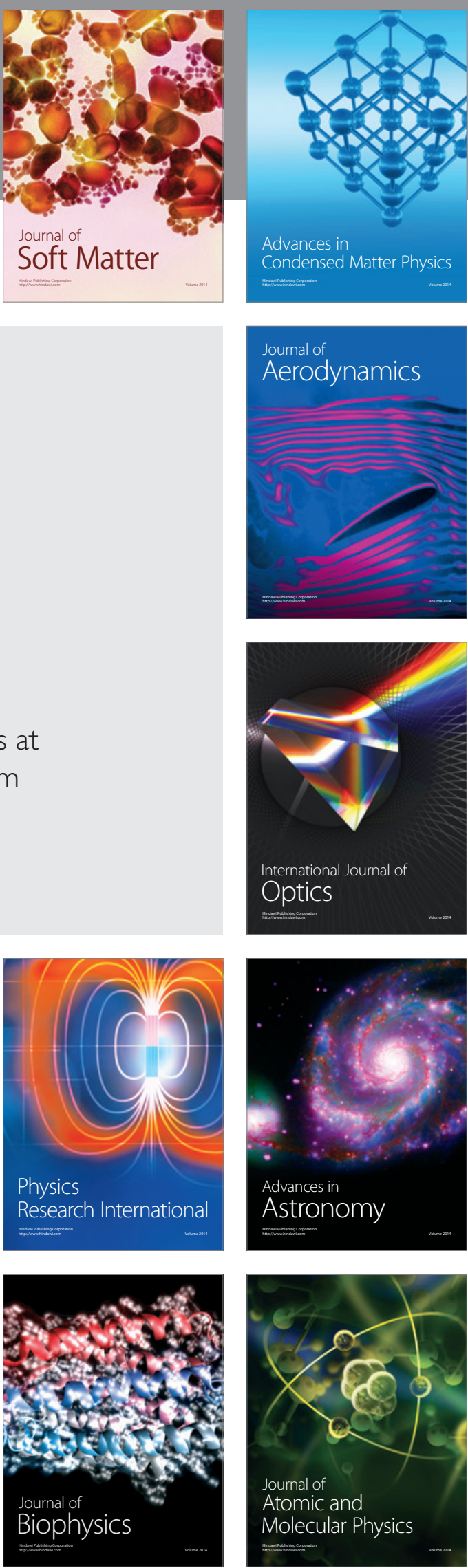